\title{
Detection of colonisation
} by extended-spectrum beta-lactamase
or carbapenemase producing Enterobacterales
from frozen stool specimens

Pisey Tan', Shweta R. Singh², Bunsoth Mao ${ }^{3}$, Konstantin Evdokimov², Vonthanak Saphonn ${ }^{3}$, Li Yang Hsu² and Paul Turner ${ }^{1,4^{*}}$ (D)

\begin{abstract}
Objective: To determine the impact of pre-culture ultra-low temperature $\left(U L T,-80^{\circ} \mathrm{C}\right)$ storage of human stool specimens on recovery of Extended-Spectrum Beta-Lactamase (ESBL) or Carbapenemase (CPM) producing Enterobacterales.

Results: Twenty stool specimens from a community-based household colonisation study in Cambodia were cultured fresh and after 4-5 days and 6 months of ULT storage (as a slurry in tryptone soya broth-10\% glycerol). Presumptive ESBL- and CPM-Escherichia coli isolates were detected in 19/20 (95\%) and 1/20 (5\%) freshly cultured specimens, respectively. The specimens yielded identical results when re-cultured after ULT storage at both time points. Detection of presumptive ESBL- and CPM-Klebsiella / Enterobacter / Citrobacter group was less frequent and slightly less stable over time. Comparison of antimicrobial susceptibility test profiles between pairs of E. coli and K. pneumoniae isolates from the two frozen culture time points revealed concordance in only 13/28 (46\%) pairs, indicating likely colonisation by multiple strains. In conclusion, ULT storage of human stool specimens prior to culture appears to be an acceptable method for managing laboratory workflow in culture-based ESBL / CPM Enterobacterales colonisation studies in high prevalence settings.
\end{abstract}

Keywords: Frozen, Storage, Detection, Faeces, Antimicrobial resistance, Escherichia coli, Klebsiella pneumoniae

\section{Introduction}

The gastro-intestinal tract is a major reservoir for antimicrobial resistant bacteria in humans. High rates of gastro-intestinal tract colonisation by extended-spectrum beta-lactamase (ESBL) and carbapenemase (CPM) producing Enterobacterales have been reported in many studies [1].

\footnotetext{
${ }^{*}$ Correspondence: pault@tropmedres.ac

${ }^{1}$ Cambodia Oxford Medical Research Unit, Angkor Hospital for Children, Siem Reap, Cambodia

Full list of author information is available at the end of the article
}

Practical coordination of laboratory work for large colonisation studies can be a challenge, especially with community-based sampling where multiple specimens may be received by the laboratory at the end of the working day. Delays to processing specimens may result in sub-optimal results. However, storage at ultra-low temperatures (ULT) with delayed processing has been well validated for certain specimen types. For example, culture of fresh or $-80{ }^{\circ} \mathrm{C}$ stored nasopharyngeal swabs collected into skim milk-tryptone soya broth-glucoseglycerol (STGG) medium results in similar detection rates of Streptococcus pneumoniae [2]. Thus, pre-culture ULT storage of swabs in STGG has become an approved 
component of the WHO pneumococcal colonisation detection protocol [3].

There has been little published on the impacts of ULT storage of stool or rectal swabs specimens in the context of cultured-based colonisation detection. Declines in total anaerobes and Bifidobacteria, but no significant change in the Enterobacteria colony counts, were identified when comparing cultures of fresh and frozen stool specimens (stored in glycerol for 4 months) from 20 infants [4]. No differences were found in total aerobe colony counts in a comparison of seven adult stool specimens cultured fresh and after storage at $-80{ }^{\circ} \mathrm{C}$ for 7 days [5]. However, there have been no evaluations specifically looking at the impact of ULT storage on detection of indicator antimicrobial resistant organisms, such as ESBL- and/or CPM-producing Escherichia coli or Klebsiella pneumoniae.

The aim of this study was to determine whether recovery of ESBL and/or CPM-producing Enterobacterales in faeces would be impacted by specimen storage at $-80^{\circ} \mathrm{C}$ prior to culture.

\section{Main text \\ Methods}

As part of a community-based study to determine colonisation prevalence of multi-drug resistant bacteria, stool specimens were collected from members of 88 households living in Siem Reap, Cambodia. All culture work was done at the clinical microbiology laboratory Angkor Hospital for Children (AHC), Siem Reap. This laboratory participates in national and international (World Health Organization Invasive Bacterial-Vaccine Preventable Diseases) external quality assurance schemes [6].

For this evaluation, 20 sequential study stool specimens from five households were processed immediately on receipt in the laboratory. Faecal slurries were prepared by emulsifying a small amount stool, picked up using a sterile swab, in $1.5 \mathrm{ml}$ of sterile tryptone soya broth (Oxoid, Basingstoke, UK) $-10 \%$ glycerol storage medium. These slurries were cultured immediately and then following 4-5 days (Frozen \#1) and 172-3 days ( 6 months, Frozen \#2) storage at $-80^{\circ} \mathrm{C}$.

For culture, $10 \mu \mathrm{L}$ of fresh or defrosted faecal slurry was streaked onto both CHROMagar ESBL, for ESBL detection, and $\mathrm{KPC}$, for CPM detection, plates (CHROMagar, Paris, France) and incubated overnight at $37{ }^{\circ} \mathrm{C}$ under aerobic conditions. The CHROMagar plates were prepared in-house following the manufacturer's instructions, including positive and negative quality control with appropriate American Type Culture Collection and inhouse bacterial strains.

From the fresh specimen culture plates, presence or absence of pink (suspected E. coli) and blue (suspected
Klebsiella sp., Enterobacter sp., Citrobacter sp.-KEC group) colonies was recorded. From the frozen specimen culture plates, colony presence or absence was recorded and then one pink and one blue colony (of the dominant morphotype, if colonial variation was noted) was picked from each plate for formal identification by matrixassisted laser desorption/ionization time of flight mass spectrometry (MALDI-ToF; VITEK MS, bioMerieux, Marcy L'Etoile, France) and antimicrobial susceptibility testing (AST) by disk diffusion. The antibiotics tested were amoxicillin-clavulanate, ampicillin, cefpodoxime, ceftazidime, ceftriaxone, chloramphenicol, ciprofloxacin, gentamicin, meropenem, nitrofurantoin, and sulphamethoxazole-trimethoprim. The 2019 version of the Clinical Laboratory Standards Institute guidelines were used to interpret AST results [7].

Differences in detection of pink or blue colonies over time was assessed using the Chi-squared for trend test, with $p$ values of $<0.05$ being considered statistically significant. For comparison of AST profiles, "intermediate" results were re-assigned as "susceptible" to give a binary readout ("susceptible" or "resistant") for each isolate-drug combination.

\section{Results}

Fresh culture of the stool specimens yielded pink colonies on 19/20 ESBL plates and 1/20 KPC plates. Blue colonies were detected on 10/20 ESBL plates and 1/20 KPC plates. Following freezing, the same pattern of pink colonies was identified on re-culture of the specimens at both time points (i.e. $100 \%$ concordance). In contrast, there was some variability in the detection of blue colonies between time points, although the differences did not reach statistical significance (Table 1). For blue colony detection on the ESBL plates, there were $15 / 20$ specimens with agreement at all three time points. In the other five, loss of blue colonies following

\begin{tabular}{|c|c|c|c|c|}
\hline \multirow[t]{2}{*}{ Time point } & \multicolumn{2}{|c|}{ Pink colonies* } & \multicolumn{2}{|c|}{ Blue colonies $^{\dagger}$} \\
\hline & ESBL plate & KPC plate & ESBL plate & KPC plate \\
\hline Fresh & $19(95)$ & $1(5)$ & $10(50)$ & $1(5)$ \\
\hline Frozen \#1 & $19(95)$ & $1(5)$ & $12(60)$ & $0(0)$ \\
\hline Frozen \#2 & $19(95)$ & $1(5)$ & $12(60)$ & $0(0)$ \\
\hline$p$ value $^{\ddagger}$ & 1.0 & 1.0 & 0.5 & 0.2 \\
\hline
\end{tabular}

*Indicative of Escherichia coli

+ Indicative of Klebsiella sp., Enterobacter sp., or Citrobacter sp. (KEC group)

‡ Chi-squared test for trend (column values) 
freezing occurred in one and blue colonies were detected only after freezing in the other four. One specimen had a blue colony on the fresh KPC plate, but this was not detected following freezing (Table 2). From frozen specimens culture plates, bacterial identity was confirmed as E. coli for all pink colonies. Of the blue colonies, 18/24 (75.0\%) were K. pneumoniae, with the other six comprising $C$. sedlakii $(\mathrm{n}=2), E$. hormaechei $(\mathrm{n}=2)$, E. kobei $(\mathrm{n}=1)$, and Enterobacter species $(\mathrm{n}=1)$.

Antimicrobial susceptibility profiles were compared for 28 pairs of E. coli $(\mathrm{n}=20)$ and $K$. pneumoniae $(\mathrm{n}=8)$ isolates (i.e. the dominant species detected), cultured from the same specimen at the two post-ULT storage culture points (Table 3 ). In 13 pairs (46.4\%), the AST profiles were identical indicating likely selection of the same strain on both cultures. In the other 15 pairs, between one and four AST results were different (median of two differences per pair). These differences were similar when comparing pairs of $E$. coli (same profile in $10 / 20,50.0 \%$ ) or K. pneumoniae (same profile in $3 / 8,37.5 \%)$.

\section{Discussion}

This pragmatic study evaluated the impact of ULT storage of faecal slurries prior to culture to detect ESBL or CPM producing Enterobacterales using commercially available chromogenic media. No significant differences in crude presumptive detection rates for these organisms were found before and after ULT storage. At the individual level, the detection of suspected ESBL- or CPM-E. coli was unaffected by ULT storage. However, there was some variability in detection of suspected ESBL- or CPM-KEC group organisms over time. Also, analysis of pairs of $E$. coli and K. pneumoniae isolates cultured from the same frozen specimen at two different time points revealed variabilities in AST profiles. The most likely explanation for this is random selection of different colonising strains, since it is known that multiple strains of drug-resistant $E$. coli and K. pneumoniae may be carried concurrently [8]. A previous study from the AHC patient population identified up to 10 discrete $E$. coli strains, as defined by multilocus sequence type, when 16 colonies were sequenced per rectal swab specimen [9]. Picking of multiple colonies per plate may have improved the chance of detection of identical strains at both time points although, similar to detection of multiple pneumococcal serotypes in

Table 2 Individual stool specimen culture summary at each time point

\begin{tabular}{|c|c|c|c|c|c|c|c|c|c|c|c|c|}
\hline \multirow[t]{3}{*}{ Specimen } & \multicolumn{6}{|c|}{ ESBL plate } & \multicolumn{6}{|c|}{ KPC plate } \\
\hline & \multicolumn{3}{|c|}{ Pink colonies } & \multicolumn{3}{|c|}{ Blue colonies } & \multicolumn{3}{|c|}{ Pink colonies } & \multicolumn{3}{|c|}{ Blue colonies } \\
\hline & Fresh & Frozen \#1 & Frozen \#2 & Fresh & Frozen \#1 & Frozen \#2 & Fresh & Frozen \#1 & Frozen \#2 & Fresh & Frozen \#1 & Frozen \#2 \\
\hline 1 & Yes & Yes & Yes & Yes & No & No & No & No & No & No & No & No \\
\hline 2 & Yes & Yes & Yes & No & No & No & No & No & No & No & No & No \\
\hline 3 & Yes & Yes & Yes & No & No & Yes & No & No & No & No & $\mathrm{No}$ & No \\
\hline 4 & Yes & Yes & Yes & No & No & No & No & No & No & No & No & No \\
\hline 5 & Yes & Yes & Yes & Yes & Yes & Yes & No & $\mathrm{No}$ & No & No & No & No \\
\hline 6 & No & No & No & No & No & No & No & $\mathrm{No}$ & No & No & No & $\mathrm{No}$ \\
\hline 7 & Yes & Yes & Yes & Yes & Yes & Yes & No & $\mathrm{No}$ & No & Yes & No & $\mathrm{No}$ \\
\hline 8 & Yes & Yes & Yes & No & No & No & No & No & No & No & No & No \\
\hline 9 & Yes & Yes & Yes & Yes & Yes & Yes & Yes & Yes & Yes & No & No & $\mathrm{No}$ \\
\hline 10 & Yes & Yes & Yes & Yes & Yes & Yes & No & No & No & No & No & No \\
\hline 11 & Yes & Yes & Yes & No & Yes & Yes & No & $\mathrm{No}$ & No & $\mathrm{No}$ & No & $\mathrm{No}$ \\
\hline 12 & Yes & Yes & Yes & No & No & No & No & No & No & No & No & No \\
\hline 13 & Yes & Yes & Yes & No & Yes & Yes & No & No & No & No & No & No \\
\hline 14 & Yes & Yes & Yes & $\mathrm{No}$ & Yes & No & No & No & No & No & No & No \\
\hline 15 & Yes & Yes & Yes & Yes & Yes & Yes & No & No & No & No & No & No \\
\hline 16 & Yes & Yes & Yes & No & No & No & $\mathrm{No}$ & No & No & $\mathrm{No}$ & No & No \\
\hline 17 & Yes & Yes & Yes & Yes & Yes & Yes & No & No & No & No & No & No \\
\hline 18 & Yes & Yes & Yes & Yes & Yes & Yes & No & No & No & $\mathrm{No}$ & No & No \\
\hline 19 & Yes & Yes & Yes & Yes & Yes & Yes & No & No & No & No & No & No \\
\hline 20 & Yes & Yes & Yes & Yes & Yes & Yes & No & No & No & No & No & No \\
\hline
\end{tabular}

"Yes" indicates detection of the colony type; "No" indicates the absence of the colony type 
Table 3 Antimicrobial susceptibility testing summary by time point in paired Escherichia coli and Klebsiella pneumoniae isolates (i.e. isolates from the same specimen cultured from frozen at two discrete time points)

\begin{tabular}{|c|c|c|c|c|}
\hline Pair ID & Organism & AST profile ${ }^{\dagger}-$ Frozen \#1 & AST profile ${ }^{\dagger}-$ Frozen \#2 & $\begin{array}{l}\text { AST profile } \\
\text { differences } \\
\text { (n) }\end{array}$ \\
\hline 1 & Escherichia coli & $----R---^{*}$ & $----R---R$ & 1 \\
\hline 2 & Escherichia coli & $--R R R^{*}--^{*}$ & $--{ }^{*} R R R--R$ & 3 \\
\hline 3 & Escherichia coli & $-R-R R---R$ & $-R-{ }^{*} R---R$ & 1 \\
\hline 4 & Escherichia coli & $----R---R$ & $----R---R$ & 0 \\
\hline 5 & Escherichia coli & $-{ }^{*}-{ }^{*} \mathrm{RR}---$ & $-\mathrm{R}-\mathrm{R} R \mathrm{R}---$ & 2 \\
\hline 6 & Klebsiella pneumoniae & $---R R---R$ & $---R R---R$ & 0 \\
\hline 7 & Escherichia coli & $----R R--R$ & $----R R--R$ & 0 \\
\hline 8 & Klebsiella pneumoniae & $---R R---R$ & $---^{* *}---{ }^{*}$ & 3 \\
\hline 9 & Escherichia coli & $-R-R R^{*}--^{*}$ & $-{ }^{*}-{ }^{*} \mathrm{RR}--\mathrm{R}$ & 4 \\
\hline 10 & Escherichia coli & $----R R--R$ & $----R R--R$ & 0 \\
\hline 11 & Klebsiella pneumoniae & $-{ }^{*}-R R-{ }^{*}-R$ & $-R-R R-R-R$ & 2 \\
\hline 12 & Escherichia coli & $R R-R R--R R$ & $R R-R R--R R$ & 0 \\
\hline 13 & Escherichia coli & $-{ }^{*}-{ }^{*} \mathrm{RR}--\mathrm{R}$ & $-R-R R R--R$ & 2 \\
\hline 14 & Klebsiella pneumoniae & $-R-R R-R-^{*}$ & $-{ }^{*}-\mathrm{RR}-{ }^{*}-\mathrm{R}$ & 3 \\
\hline 15 & Escherichia coli & $-R R R R R--R$ & $-R R R R R--R$ & 0 \\
\hline 16 & Escherichia coli & $---R R----$ & $---{ }^{*} \mathrm{R}----$ & 1 \\
\hline 17 & Escherichia coli & $--R R R R--R$ & $--R R R R--R$ & 0 \\
\hline 18 & Escherichia coli & $---\mathrm{RR}----$ & $---\mathrm{RR}----$ & 0 \\
\hline 19 & Escherichia coli & $-{ }^{*}-\mathrm{RRR}---$ & $-R-{ }^{*} R^{*}---$ & 3 \\
\hline 20 & Klebsiella pneumoniae & $-R-R R R^{*}-R$ & $-R-R R^{*} R-R$ & 2 \\
\hline 21 & Escherichia coli & $---R R---R$ & $---R R---R$ & 0 \\
\hline 22 & Escherichia coli & - RRRR $---^{*}$ & $-{ }^{*} R * R---R$ & 3 \\
\hline 23 & Escherichia coli & $---R R---R$ & $---R R---R$ & 0 \\
\hline 24 & Klebsiella pneumoniae & $---R R---R$ & $---{ }^{*} R---^{*}$ & 2 \\
\hline 25 & Escherichia coli & $--R R R---R$ & $--R * R---R$ & 1 \\
\hline 26 & Klebsiella pneumoniae & $-R-R R R--R$ & $-R-R R R--R$ & 0 \\
\hline 27 & Escherichia coli & $---R R---R$ & $---R R---R$ & 0 \\
\hline 28 & Klebsiella pneumoniae & $-R--R----$ & $-R--R----$ & 0 \\
\hline
\end{tabular}

${ }^{\dagger}$ The antimicrobial susceptibility (AST) profile consists of a " - " (susceptible / intermediate) or "R" (resistant) for each of the following antimicrobials (in order): amoxicillin-clavulanate, ceftazidime, chloramphenicol, ciprofloxacin, cefpodoxime, ceftriaxone, gentamicin, nitrofurantoin, meropenem, and sulphamethoxazoletrimethoprim. Mismatched susceptibility results within an isolate pair are labelled with asterisks "*". Ampicillin and cefpodoxime results were excluded as they were "R" throughout

nasopharyngeal swab specimens, this would dependent on the strain relative abundances [10].

In conclusion, ULT storage of faecal slurries for up to 6 months prior to culture appears to be an acceptable method for managing laboratory workflow in culturebased ESBL / CPM E. coli, and probably other Enterobacterales, colonisation studies in high prevalence settings.

\section{Limitations}

The major limitation of the study is that colony densities were not recorded to determine whether yield of target organisms decreased over prolonged storage. Also, the prevalence of ESBL colonisation was found to be extremely high in the study population so results may not be transferable to studies conducted in populations with lower colonisation prevalence. Given the identification of target species with different AST profiles over time, further work is required to characterise the longitudinal stability of multiple colonising clones at $-80{ }^{\circ} \mathrm{C}$. It may be that all clones are not equally stable during storage, either due to clone-specific factors or population density in the original specimen, leading to differences in recovery over time. Metagenomic sequencing based experiments would best determine the impact of ULT storage on relative abundance of specific clones over time and would help to determine if there is a "best before" date for culture of stored faecal specimens. Finally, given the slight variability in detection of KEC group organisms over time, a 
larger study may be warranted to confirm our findings for species other than E. coli.

\begin{abstract}
Abbreviations
AHC: Angkor Hospital for Children; AST: Antimicrobial susceptibility test(ing); CPM: Carbapenemase; ESBL: Extended spectrum beta-lactamase; KEC: Klebsiella Sp.—Enterobacter sp.—Citrobacter sp.; KPC: Klebsiella pneumoniae Carbapenemase; MALDI-ToF: Matrix-assisted laser desorption/ionization time of flight mass spectrometry; STGG: Skim milk—tryptone soya broth-glucose-glycerol; ULT: Ultra-low temperature.
\end{abstract}

\section{Acknowledgements}

The authors are grateful to all study participants and the study data collection and laboratory teams.

\section{Authors' contributions}

PT2, SRS, BM, KE, VS, LYS conceived the study. BM, SRS supervised collection of specimens. PT1 performed the laboratory experiments. PT1 and PT2 drafted the manuscript. All authors read and approved the final manuscript.

\section{Funding}

Not externally funded.

\section{Availability of data and materials}

The datasets generated and/or analysed during the current study are available in the Figshare repository, https://figshare.com/articles/dataset/COMRU-NUSUHS_ESBL-CPM-E_stool_data/12915842.

\section{Ethics approval and consent to participate}

The study protocol was approved by the National Ethics Committee for Health Research, Cambodia (148 NECHR), National University of Singapore Institutional Review Board (H-18-069), and the University of Oxford Tropical Research Ethics Committee (OxTREC 551-18). All participants, or their legally acceptable representative, gave written informed consent prior to study enrolment.

\section{Consent for publication}

Not applicable.

\section{Competing interests}

The authors have no competing interests to declare.

\section{Author details}

${ }^{1}$ Cambodia Oxford Medical Research Unit, Angkor Hospital for Children, Siem Reap, Cambodia. ${ }^{2}$ Saw Swee Hock School of Public Health, National University of Singapore, Singapore, Singapore. ${ }^{3}$ University of Health Sciences, Phnom Penh, Cambodia. ${ }^{4}$ Centre for Tropical Medicine and Global Health, Nuffield Department of Clinical Medicine, University of Oxford, Oxford, UK.
Received: 27 May 2020 Accepted: 9 September 2020

Published online: 14 September 2020

\section{References}

1. Karanika S, Karantanos T, Arvanitis M, Grigoras C, Mylonakis E. Fecal colonization with extended-spectrum beta-lactamase-producing enterobacteriaceae and risk factors among healthy individuals: a systematic review and metaanalysis. Clin Infect Dis. 2016;63(3):310-8.

2. O'Brien KL, Bronsdon MA, Dagan R, Yagupsky P, Janco J, Elliott J, et al. Evaluation of a medium (STGG) for transport and optimal recovery of Streptococcus pneumoniae from nasopharyngeal secretions collected during field studies. J Clin Microbiol. 2001;39(3):1021-4.

3. Satzke C, Turner P, Virolainen-Julkunen A, Adrian PV, Antonio M, Hare $\mathrm{KM}$, et al. Standard method for detecting upper respiratory carriage of Streptococcus pneumoniae: updated recommendations from the World Health Organization Pneumococcal Carriage Working Group. Vaccine. 2013;32(1):165-79.

4. Guerin-Dana C, Andreieux C, Szylit O. Storage of intestinal bacteria in samples frozen with glycerol. Microb Ecol Health Dis. 1999;11:180-2.

5. Fouhy F, Deane J, Rea MC, O'Sullivan O, Ross RP, O'Callaghan G, et al. The effects of freezing on faecal microbiota as determined using MiSeq sequencing and culture-based investigations. PLOS ONE. 2015;10(3):e0119355.

6. Fox-Lewis A, Takata J, Miliya T, Lubell Y, Soeng S, Sar P, et al. Antimicrobial Resistance in Invasive Bacterial Infections in Hospitalized Children, Cambodia, 2007-2016. Emerg Infect Dis. 2018;24(5):841-51.

7. CLSI. Performance standards for antimicrobial susceptibility testing. CLSI document M100-S29. 29th ed. Wayne: Clinical and Laboratory Standards Institute; 2019.

8. van Aartsen JJ, Moore CE, Parry CM, Turner P, Phot N, Mao S, et al. Epidemiology of paediatric gastrointestinal colonisation by extended spectrum cephalosporin-resistant Escherichia coli and Klebsiella pneumoniae isolates in north-west Cambodia. BMC Microbiol. 2019;19(1):59.

9. Stoesser N, Sheppard AE, Moore CE, GolubchikT, Parry CM, Nget P, et al. Extensive within-host diversity in fecally carried extended-spectrum-beta-lactamase-producing Escherichia coli isolates: implications for transmission analyses. J Clin Microbiol. 2015;53(7):2122-31.

10. Huebner RE, Dagan R, Porath N, Wasas AD, Klugman KP. Lack of utility of serotyping multiple colonies for detection of simultaneous nasopharyngeal carriage of different pneumococcal serotypes. Pediatr Infect Dis J. 2000;19(10):1017-20.

\section{Publisher's Note}

Springer Nature remains neutral with regard to jurisdictional claims in published maps and institutional affiliations.
Ready to submit your research? Choose BMC and benefit from:

- fast, convenient online submission

- thorough peer review by experienced researchers in your field

- rapid publication on acceptance

- support for research data, including large and complex data types

- gold Open Access which fosters wider collaboration and increased citations

- maximum visibility for your research: over 100M website views per year

At BMC, research is always in progress.

Learn more biomedcentral.com/submissions 